\title{
MR Angiographic-Guided Percutaneous Sclerotherapy for Venous Vascular Malformations: A Radiation Dose-Reduction Strategy
}

\author{
S.G. Imbesi, D.A. Green, A. Cho, and R.S. Pakbaz
}

\begin{abstract}
SUMMARY: We present a new technique using MRA instead of the usual DSA to provide guidance in the treatment of venous vascular malformations. When one performs this embolization procedure, appropriate needle positioning within the malformation must be confirmed before injection of the sclerosing agent to prevent untoward complications. Time-resolved imaging of contrast kinetics-MRA can accurately depict the angioarchitecture of the lesion, which substantially reduces the total radiation dose in these patients who are commonly in the pediatric age group and usually require numerous treatment episodes.
\end{abstract}

$V^{2}$ enous malformations are the most common vascular malformation and can be found throughout the body but tend to be located in the head and neck region or the extremities. ${ }^{1-3}$ They are low-flow lesions of an abnormal venous network of dilated vascular spaces consisting of thin-walled sponge-like channels of varying size with adventitial fibrosis, sparse clumped smooth-muscle cells, internal regions of thrombosis, and occasional phleboliths. On examination, venous malformations are compressible, nonpulsatile masses, usually exhibiting a bluish tint of the overlying skin. Provocative maneuvers such as Valsalva can show enlargement of the lesion. They present in childhood; mostly enlarge with time, especially during puberty and pregnancy due to hormonal effects; and do not regress. Thus, these lesions may require treatment. Accurate diagnosis is required to confirm the nature of the vascular malformation so that appropriate treatment can be planned.

Mulliken and Glowacki ${ }^{4}$ differentiated vascular anomalies as either vascular neoplasms or vascular malformations. The vascular neoplasms (or true hemangiomas) show endothelial hyperplasia, while the vascular malformations, being an error of embryologic development, show normal endothelial turnover. This scheme was further subclassified by the International Society for the Study of Vascular Anomalies, which divided the vascular malformations between simple (capillary, venous, or lymphatic malformations) or combined (arteriovenous malformation, arterio-

Received March 17, 2015; accepted after revision June 24.

From the Department of Radiology (S.G.I., D.A.G., A.C.), University of California, San Diego Health System, San Diego, California; and Department of Radiology (R.S.P.), VA San Diego Healthcare System, San Diego, California.

Please address correspondence to Steven G. Imbesi, MD, Department of Radiology, University of California, San Diego Health System, 200 West Arbor Dr, Mail Code \#8756, San Diego, CA 92103; e-mail: simbesi@ucsd.edu

http://dx.doi.org/10.3174/ajnr.A4518 venous fistula, and capillary and/or venous and/or lymphatic malformation) forms. ${ }^{5}$ Finally, vascular malformations can also be categorized as either low-flow (capillary, venous, lymphatic, or any combination of these) or high-flow (arteriovenous malformation, arteriovenous fistula). On imaging, Doppler sonography can be used to show the low-flow profile of the venous malformation. On CT, a heterogeneous lesion is usually seen with fat and calcified phleboliths easily identifiable. The lesion enhances slowly and peripherally after contrast administration. MR imaging is best to delineate the lesion margins and detect invasion into surrounding structures, with T2-weighted STIR imaging showing the mostly bright-blood-filled channels.

Patient symptoms are related to lesion size and location. While venous malformations are noted in the skin and adjacent subcutaneous tissues, they usually also involve the underlying muscle, bone, and other tissues. Thrombosis, bleeding, and swelling can lead to pain. Lesions of the head and neck can present aesthetic issues for the patient but can also distort speech and even obstruct the upper airway. In particular in these instances, treatment of the venous malformation is necessary. This is performed via direct needle puncture of the lesion. Rapid blood return from the needle hub assumes correct needle positioning within the lesion; however, a phlebogram must be obtained (presently via digital subtraction angiography) to confirm the exact needle localization (Fig 1). A sclerosing agent such as absolute ethanol or sodium tetradecyl sulfate is then injected. The confirmation of correct needle positioning is mandatory because extravasation of the sclerosant can lead to direct tissue necrosis or nerve injury. Observation of large draining veins may also preclude treatment because the peripheral extent of the sclerosant into the systemic circulation may lead to hemolysis or cardiac/pulmonary/renal complications. We propose MR angiography-time-resolved imaging of 


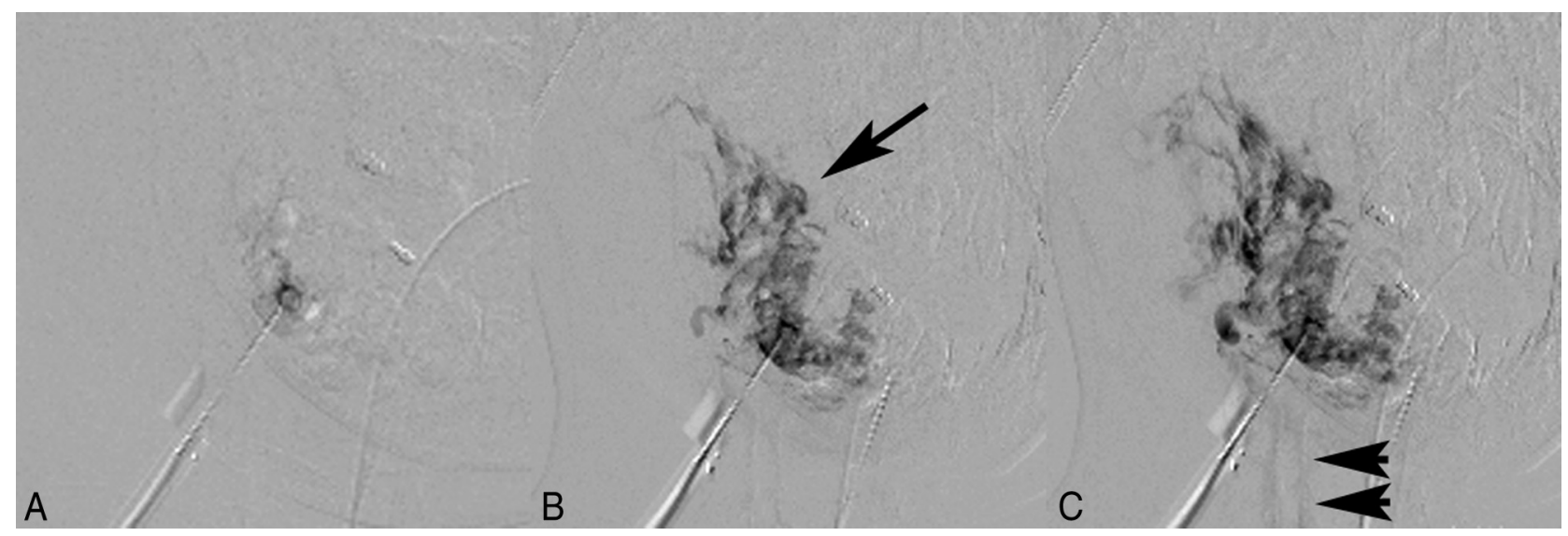

FIG 1. Early $(A)$, mid $(B)$, and late $(C)$ phase direct needle puncture phlebography of a left facial venous malformation by using a traditional digital subtraction angiography technique shows appropriate needle localization for subsequent embolization. There is satisfactory sequential opacification of the venous channels of the lesion (arrow) and only faint flow into small draining veins (arrowheads), with no evidence of contrast extravasation or demonstration of large draining veins.

contrast kinetics to obtain appropriate needle positioning and depict the angioarchitecture of the venous malformation to decrease the radiation dose when treating these lesions.

\section{Case Series}

In 5 patients, including 4 with venous malformations of the head/ neck and 1 with a peripheral limb venous malformation of the knee, 14 sclerotherapy sessions have been performed. A standard 1.5T superconducting magnet was used for all the procedures. The study was approved by the local institutional review board. The patients were placed under general anesthesia and prepped and draped in the usual sterile manner in the MR imaging suite, and sterile covers were placed over the MR imaging coils that were eventually positioned over the ROI. Initial multiplanar, multisequence MR images were obtained to assess the lesion. Twenty-two-gauge titanium needles were then inserted through the skin for direct puncture access to the venous malformation. Along the tubing adjacent to the needle, a 3-way stopcock was also placed to allow attachment of a second shorter tubing that is connected to a syringe prefilled with contrast material. Gadobenate dimeglumine (MultiHance; Bracco Diagnostics, Princeton, New Jersey) in a 1:100 dilution with normal saline solution was used for the contrast administration. Approximately $1.0-1.5 \mathrm{~mL}$ of contrast material was preloaded into the needle and the most distal portion of the tubing beyond the stopcock. The stopcock was then opened to the long connecting tubing so that injection could be performed by the operator outside the bore of the magnet.

Once the patients were positioned within the magnet bore, continuous MRA- time-resolved imaging of contrast kinetics was obtained before and during the injection of the $1.0-1.5 \mathrm{~mL}$ of contrast medium followed by $10 \mathrm{~mL}$ of normal saline solution flush. The parameters of the image acquisition were as follows: sagittal plane slab orientation with 10 sections by using TR, 4.028 milliseconds; TE, 1.672 milliseconds; NEX, 0.5; section thickness, $2.6 \mathrm{~mm}$; FOV, $220 \times 220 \mathrm{~mm}$; matrix, $256 \times 192$, echo-train, 1 ; flip angle, $30^{\circ}$; bandwidth, 488.3 hertz. The precontrast images (13-second acquisition) were used as a mask and were digitally subtracted from the postcontrast images (51-second acquisition). The sequential images were then viewed as a cine loop to demonstrate intralesional vascular flow with time. The chosen parame- ters result in a relatively fast 1.8 seconds per frame for the cine loop, which also achieves reasonable spatial resolution that enables appropriate evaluation of the venous vascular flow. Finally, summation maximum-intensity-projection images can also be viewed and $3 \mathrm{D}$ rotated to observe the entirety of the opacification of the vascular lesion and venous runoff altogether in a single slab. If a satisfactory needle position was achieved, the sclerosant ( $a b-$ solute ethanol, $0.1 \mathrm{~mL} / \mathrm{Kg}$ body weight) was then injected. Following a dwell time of 10 minutes, the contrast injection was repeated and the result of embolization was observed (Figs 2-4).

Following completion of the sclerotherapy session, the patient was closely observed with airway management (in particular for the head/neck embolizations) and placed on methylprednisolone corticosteroids for reduction of potential subsequent soft-tissue swelling and given hydrocodone and acetaminophen for pain control. Excellent response to therapy was achieved in every case, evidenced by diminished flow within the lesions and pruning of the lesion vasculature. No procedural complications were encountered, and all patients had an uneventful postprocedural recovery.

\section{DISCUSSION}

When treatment of venous vascular malformations is required due to lesion bleeding, pain, mass effect, or esthetic problems, an angiogram must be obtained to confirm appropriate needle positioning within the lesion before injection of the sclerosing agent to prevent complications and untoward effects of the procedure. ${ }^{6,7}$ This information is traditionally obtained by using digital subtraction angiography. While the radiation dose is relatively low for each individual treatment episode, in many instances, numerous treatment sessions are usually required due to the intrinsic multiloculated, trans-spatial nature of the lesion. In addition, only a small amount of sclerosant (in particular absolute ethanol) can be delivered to the tissues in a given treatment session to limit systemic toxicity. Thus, the total accumulated radiation dose to achieve complete lesion obliteration can become rather substantial. Additionally, because these lesions commonly occur in the head and neck region, adjacent very radiosensitive structures such as the thyroid gland and ocular lenses are frequently in the field of 


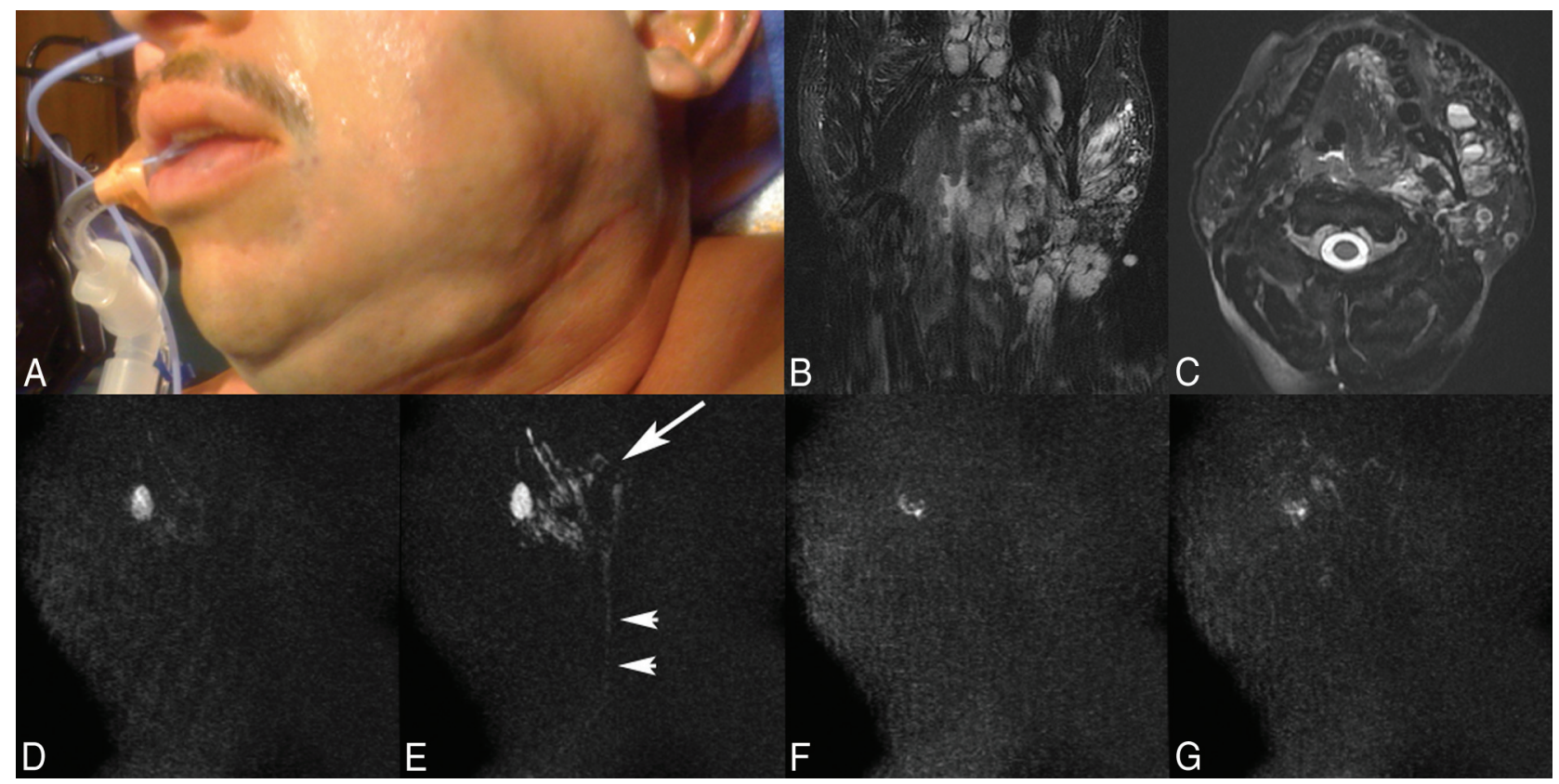

FIG 2. A 36-year-old man with a left facial venous malformation who undergone 14 treatments during the past 3 years, the last 2 with MR imaging guidance. Overview of the direct-injection MR angiography technique for embolization of the lesion. $A$, Patient photograph before MR sclerotherapy shows left facial swelling from a persistent left facial venous malformation. Coronal (B) and axial (C) T2-weighted fat-saturated MR images demonstrate the typical imaging characteristics of a venous vascular malformation, including a mixed signal intensity but mostly bright trans-spatial lesion extending both superficial and deep to the left mandible composed of multiloculated blood-filled channels with fluid-fluid levels producing local mass effect. Pretreatment MR angiograms, early $(D)$ and late $(E)$ phase, show appropriate needle positioning within the loculated blood-filled channels of the venous malformation (arrow) with slow venous runoff into a small draining vein (arrowheads). Posttreatment MR angiograms, early $(F)$ and late $(G)$ phase, demonstrate pruning of the vascular channels of the venous malformation with markedly diminished flow into the draining vein, compatible with satisfactory embolization of the lesion.

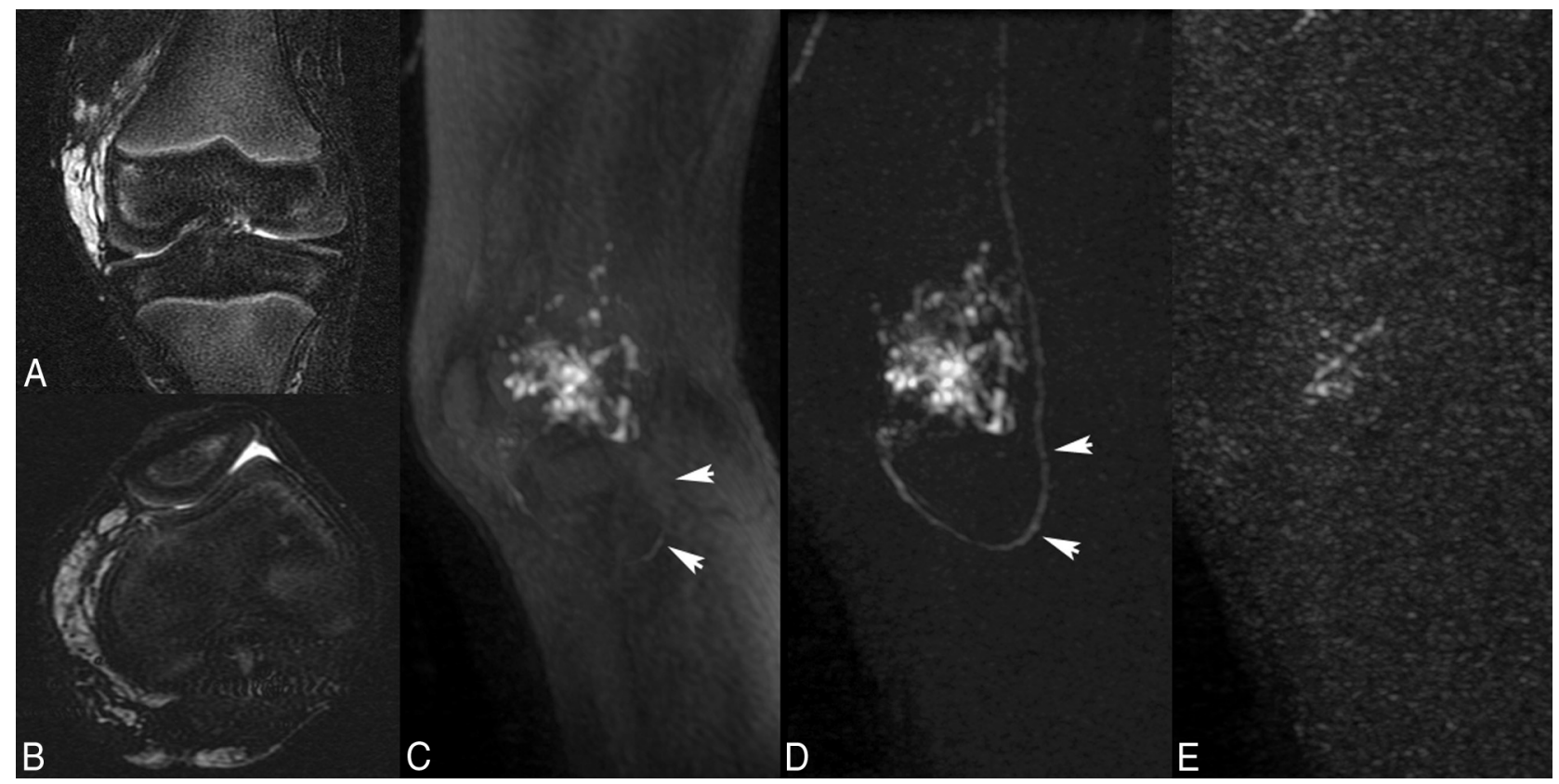

FIG 3. A 6-year-old boy with a peripheral venous malformation of the left knee. Note depiction of the mask subtraction technique that results in more complete visualization of the vascular structures on MR angiography. Coronal $(A)$ and axial $(B)$ T2-weighted fat-saturated MR images demonstrate a multiloculated increased T2-signal venous vascular malformation in the left knee, particularly surrounding the medial and posterior portions of the distal femur. Pretreatment MR angiogram, without $(C)$ and with $(D)$ digital mask subtraction, shows opacification of the venous malformation but limited visualization of the draining vein (arrowheads) without mask subtraction; however, there is not only improved visualization of the venous malformation but also excellent visualization of the draining vein (arrowheads) achieved following mask subtraction of the images. E, Posttreatment MR angiogram again shows pruning of the vascular channels of the venous malformation with no significant flow into the draining vein, compatible with satisfactory embolization of the lesion. 


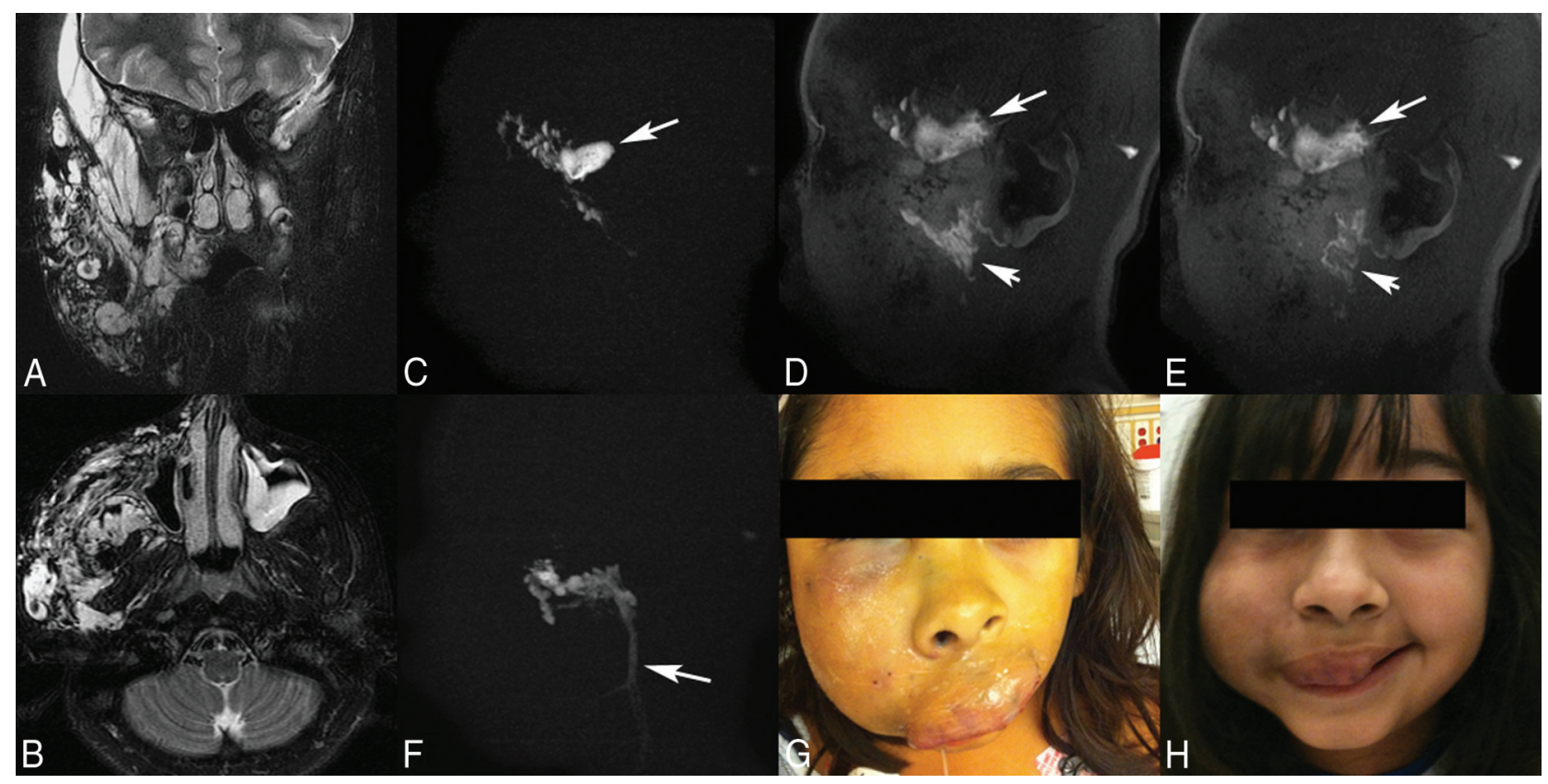

FIG 4. A 10 -year-old girl with a very large venous malformation of the right face, who has had $>20$ previous treatment episodes with traditional digital subtraction angiography guidance, now being treated with MR imaging guidance. These images demonstrate the necessity of angiography to assure proper needle positioning. Coronal $(A)$ and axial $(B)$ T2-weighted fat-saturated MR images show the very large venous vascular malformation extending essentially throughout all superficial and deep spaces of the right face, producing significant local mass effect. C, Pretreatment MR angiogram, initial needle placement, demonstrates opacification of the venous malformation but also a focus of stagnation of contrast material that remained on the late-phase images, indicating extravasation (arrow). Thus, this is not a safe location to treat, and sclerotherapy was not performed. Note that normal venous backflow from the needle hub was initially detected, but adequate needle placement can only be assured following angiography. Early $(D)$ and late $(E)$ phase pretreatment MR angiograms, second needle placement, again demonstrate opacification of the venous malformation but now also show runoff of the contrast material on the late-phase image, indicating safe needle placement for embolization (arrowheads). Note the continued presence of the previously extravasated contrast material from the first injection on this nonmask subtracted image (arrows). F, Early-phase pretreatment MR angiogram, third needle placement, depicts needle localization within the venous malformation; however, there is very rapid filling of an enlarged draining vein (arrow). Thus, this is not a safe location to treat, and sclerotherapy was not performed. Again, adequate needle placement can only be assured following angiographic visualization of the lesion. Patient photographs before $(G)$ and following $(H)$ MR sclerotherapy show significant improvement in right facial swelling; however, a persistent right facial venous malformation is still present, requiring additional multiple future treatment sessions.

treatment and thus receive overall large doses of radiation. Finally, these lesions usually occur in the pediatric population, warranting further concern regarding the total radiation dose given to the patient, because the longer subsequent life span of these young patients markedly increases the potential for future development of neoplastic disease.

For example, regarding radiation-induced thyroid cancer, the following can be surmised: The excess relative risk of thyroid cancer per sievert of $\mathrm{x}$-ray radiation is $10 \times$ that of baseline for patients $0-9$ years of age, $3 \times$ baseline for patients $10-19$ years, and $0.3 \times$ baseline for patients $20-39$ years. ${ }^{8-11}$ Thus, while the excess relative risk is not that significant for adults, it becomes very significant for the pediatric population because the pediatric risk is $10-30 \times$ that of the adult. One minute of fluoroscopy yields approximately $3-\mathrm{mSv} \mathrm{x}$-ray radiation effective dose to the thyroid gland, which is mostly due to internal scatter and therefore cannot be further reduced, even with dose-reduction techniques, including tight collimation or thyroid shielding (without thyroid shielding, the dose can be as high as $10 \mathrm{mSv} / \mathrm{min}$ ). Of note, $3 \mathrm{mSv}$ is equivalent to 1 year of background radiation or approximately 30 chest radiographs.

The average procedural fluoroscopy time for each venous malformation sclerotherapy treatment session at our institution is usually 1.5 minutes (including dose-reduction techniques such as pulsed fluoroscopy and reduced frame rate). Therefore, each procedure results in approximately $0.005-\mathrm{Sv}$ radiation exposure to the thyroid gland, leading to a pediatric excess relative risk of 0.05 above baseline. Our patient (Fig 4), who has undergone $>20$ prior treatment sessions, now has an excess relative risk of 1.0, which is $2 \times$ the natural risk of the disease. Because the lifetime natural risk of thyroid cancer in a young female is $1.5 \%$, her risk is now $3 \%$, assuming that all her procedures were performed with thyroid shielding. Without thyroid shielding, her risk could be as high as $10 \%$, and because her lesion is still not fully resolved, she will require numerous additional treatment sessions. This risk underscores the need to reduce the radiation dose as much as possible, especially in the pediatric population, and exemplifies the ongoing effort of the Alliance for Radiation Safety in Pediatric Imaging with their "Image Gently" campaign and the joint effort of the American College of Radiology, Radiological Society of North America, American Society of Radiologic Technologists, and the American Association of Physicists in Medicine with their "Image Wisely" campaign. ${ }^{12-14}$

We have developed a method to completely eliminate digital subtraction angiography $\mathrm{x}$-ray radiation exposure during treatment of venous vascular malformations. Using MR imaging, angiographic images of the venous malformation can be obtained to eventually directly guide sclerotherapy of the lesion. Angiography 
is achieved by direct needle puncture into the malformation with subsequent injection of very dilute gadolinium contrast agent during MR imaging. The time-resolved imaging technique produces an angiographic "runoff" observation of the venous malformation and the draining venous system. Lack of contrast extravasation into the adjacent soft tissues and lack of rapid flow into large draining veins confirms appropriate needle localization and allows safe embolization of the lesion. Following embolization, repeat contrast injection can show efficacy of the treatment by demonstrating pruning of the venous malformation vasculature and slowing of flow into the draining veins. The needle is then repositioned into another region of the lesion, and the process can be repeated. To our knowledge, this is the first time MR angiographic guidance has been used to completely supplant digital subtraction angiography in the real-time evaluation of angiographic vascular flow during active neurovascular embolization. Obviously, this new technique fully exemplifies the principle and radiologists' duty to obtain a radiation dose as low as reasonably achievable by completely eliminating radiation exposure for this procedure. The lack of ionizing radiation by using this technique is extremely promising for the future development of additional applications in the neurointerventional armamentarium, especially when treating pediatric patients who are most susceptible to the harmful effects of $\mathrm{x}$-ray radiation.

Limitations of the technique include extended use of the MR imaging system and an angiographic frame rate of approximately 1.8 seconds. With regard to the extended time in the MR imaging suite, experience performing the procedure has reduced the initial time of a treatment session from 2 hours to 1 hour. This reduction has been achieved by further streamlining the process, including patient and technologist education, staff familiarity with the examination proto$\mathrm{col}$, and placement of multiple needles simultaneously into different portions of the lesion for rapid transition from one treatment site to the next. While the current technology only provides an MR angiographic temporal resolution of 1.8 seconds/frame to maintain the necessary detailed spatial resolution to clearly evaluate the lesion, this is sufficient to visualize venous flow and allows safe embolization of venous vascular malformations. Although this is slow compared with the current DSA temporal resolution, as improvements in magnet performance and image acquisition software continue, more rapid frame rates may one day result in the ability to adequately visualize detailed arterial flow, with the promise of performing many other types of embolization with this technique. Finally, metallic objects such as braces on the patient's teeth (common in the pediatric age group) can result in marked susceptibility artifacts preventing visualization of the lesion on MR imaging and precluding MR imagingguided embolization.

However, an additional benefit of the technique is the ability to acquire pre-embolization MR images of the lesion, either for de novo lesion evaluation or follow-up assessment of the prior sclerotherapy session, saving the patient time and expense because this 1 technique can now be used for both lesion visualization and lesion treatment in a single patient visit/setting. To date, 5 patients at our institution (4 with venous malformations of the head/neck and 1 with peripheral limb venous malformation of the knee) with a total of 14 sclerotherapy sessions have been treated with this technique. Excellent response to therapy was achieved in every case, evidenced by dimin- ished flow within the lesions and pruning of the lesion vasculature. No procedural complications were encountered, and all patients had an uneventful postprocedural recovery.

\section{CONCLUSIONS}

MRA-direct-puncture time-resolved imaging of contrast kinetics for guidance during venous malformation sclerotherapy allows adequate lesion visualization and sufficient temporal resolution to assess vascular flow and evaluate treatment results. The obvious benefit of this technique is that no additional radiation is necessary to treat these lesions, which typically occur in the pediatric population and usually require numerous treatment sessions, resulting in significant reduction in total radiation dose. Limitations of this technique include a low temporal resolution compared with conventional digital subtraction angiography and high use of the MR imaging during relatively long procedural times. In the future, continued optimization of the technique will improve temporal resolution and increase patient throughput.

\section{REFERENCES}

1. Dubois J, Garel L. Imaging and therapeutic approach of hemangiomas and vascular malformations in the pediatric age group. Pediatr Radiol 1999;29:879-93 CrossRef Medline

2. Dubois J, Soulez G, Oliva VL, et al. Soft-tissue venous malformations in adult patients: imaging and therapeutic issues. Radiographics 2001;21:1519-31 CrossRef Medline

3. Navarro OM, Laffan EE, Ngan BY. Pediatric soft-tissue tumors and pseudo-tumors: MR imaging features with pathologic correlation, part 1-imaging approach, pseudotumors, vascular lesions, and adipocytic tumors. Radiographics 2009;29:887-906 CrossRef Medline

4. Mulliken JB, Glowacki J. Hemangiomas and vascular malformations in infants and children: a classification based on endothelial characteristics. Plast Reconstr Surg 1982;69:412-22 CrossRef Medline

5. Dasgupta R, Fishman SJ. ISSVA classification. Semin Pediatr Surg 2014;23:158-61 CrossRef Medline

6. Hyodoh H, Hori M, Akiba $H$, et al. Peripheral vascular malformations: imaging, treatment approaches, and therapeutic issues. Radiographics 2005;25:S159-71 CrossRef Medline

7. Goyal M, Causer PA, Armstrong D. Venous vascular malformations in pediatric patients: comparison of results of alcohol sclerotherapy with proposed MR imaging classification. Radiology 2002;223: 639-44 CrossRef Medline

8. Ron E, Lubin JH, Shore RE, et al. Thyroid cancer after exposure to external radiation: a pooled analysis of seven studies. Radiat Res 1995;141:259-77 CrossRef Medline

9. Ron E. Cancer risks from medical radiation. Health Phys 2003;85: 47-59 CrossRef Medline

10. Kleinerman RA. Cancer risks following diagnostic and therapeutic radiation exposure in children. Pediatr Radiol 2006;36(suppl 2): 121-25 CrossRef Medline

11. Sinnott B, Ron E, Schneider AB. Exposing the thyroid to radiation: a review of its current extent, risks, and implications. Endocr Rev 2010;31:756-73 CrossRef Medline

12. Farman AG. Image gently: enhancing radiation protection during pediatric imaging. Oral Surg Oral Med Oral Pathol Oral Radiol 2014; 117:657-58 CrossRef Medline

13. Brink JA, Amis ES Jr. Image Wisely: a campaign to increase awareness about adult radiation protection. Radiology 2010;257:601-02 CrossRef Medline

14. Goske MJ, Frush DP, Brink JA, et al. Curbing potential radiationinduced cancer risks in oncologic imaging: perspectives from the "image gently" and "image wisely" campaigns. Oncology (Williston Park) 2014;28:232-38, 243 Medline 